\title{
Gerontotecnologia para prevenção de quedas: cuidado de enfermagem ao idoso com Parkinson
}

\author{
Gerontotechnology for fall prevention: nursing care for older adults with Parkinson \\ Gerontecnología para la prevención de caídas: cuidados de enfermería \\ en adultos mayores con la enfermedad de Parkinson
}

Como citar este artigo:

Ferreira JM, Hammerschmidt KSA, Heideman ITSB, Alvarez AM, Santos SMA, Fabrizzio GC. Gerontotechnology for fall prevention: nursing care for older adults with Parkinson. Rev Esc Enferm USP. 2021;55:e03748. https://doi.org/10.1590/S1980-220X2020018403748

\section{Juliana Martins Ferreira ${ }^{1}$ \\ Karina Silveira de Almeida Hammerschmidt ${ }^{1}$}

Ivonete Teresinha Schulter Buss Heideman $^{1}$

\section{Angela Maria Alvarez ${ }^{1}$ \\ Silvia Maria Azevedo dos Santos ${ }^{1}$ \\ Greici Capellari Fabrizzio ${ }^{1}$}

${ }^{1}$ Universidade Federal de Santa Catarina, Programa de Pós-Graduação em

Enfermagem, Florianópolis, SC, Brasil.

\begin{abstract}
Objective: To assess the contribution of gerontotechnologies in nursing care to older adults with Parkinson's disease, aiming at the prevention of falls. Method: Convergent Assistance Research, through the development and evaluation of gerontechnologies focused on the prevention of falls in older adults with Parkinson's Disease. Clinical evaluation, interviews and workshops with older adults were carried out, as well as analysis and judgment of the materials developed by ten judges certified in gerontology by the Brazilian Society of Geriatrics and Gerontology. Results: Older adults need at least six years of study to understand the educational gerontotechnology development. The objectives related to the content, understanding of the text, illustration, presentation, motivation and cultural adaptation were achieved. Conclusion: The educational gerontotechnology developed has relevant content, which can be used by older adults, family members and caregivers, to generate clarification of questions on the theme of fall prevention for older adults with Parkinson's Disease.
\end{abstract}

DESCRIPTORS

Multimedia; Nursing Care; Aged; Parkinson Disease. 


\section{INTRODUÇÃO}

A prática educativa é tarefa inerente ao enfermeiro, que pode estimular o idoso a refletir sobre sua rotina e condutas, assumindo papel transformador no processo saúde e doença. Ao encontro dessa prática e incentivo ao autocuidado, a gerontotecnologia pode ser utilizada como estratégia, sendo considerada um amplo campo das tecnologias educacionais direcionadas aos idosos, que envolve a aplicação dos estudos da gerontologia, a fim de desenvolver e difundir objetos de cunho tecnológico. Além disso, são ferramentas capazes de fornecer informação, favorecer a comunicação, criar novas perspectivas de tecnologias para apoio aos idosos e fortalecer as ações de cuidado do enfermeiro ${ }^{(1-3)}$.

A proposta da gerontotecnologia converge para um cenário de envelhecimento populacional mundial, pois, de acordo com Organização Mundial da Saúde ${ }^{(4)}$, para 2020, espera-se que existam no mundo 1,1 bilhões de idosos, sendo que esse número passará para 3,1 bilhões em 2100. Nesse cenário, para a população idosa brasileira, estima-se que será de 29,9 milhões em 2020, alcançando 72,4 milhões em 2100.

Com o aumento da população idosa, faz-se necessário o desenvolvimento de produtos e serviços que atendam às necessidades e capacidades desse público ${ }^{(5)}$. O fenômeno natural do processo de envelhecimento implica alterações importantes na condição de saúde do idoso com o declínio da função fisiológica e a predisposição para múltiplas patologias associadas às doenças crônicas não transmissíveis (DCNT), visto que estão entre as principais doenças que acometem os idosos $^{(5-6)}$.

Dentre as DCNT, destaca-se a Doença de Parkinson (DP) como a segunda patologia neurodegenerativa mais prevalente em pessoas acima dos 50 anos, cuja incidência aumenta com o envelhecimento. Os sinais característicos da patologia são tremor em repouso, rigidez, bradicinesia, e alterações de postura, marcha e instabilidade postural ${ }^{(7)}$.

Com o avanço da DP e uso prolongado da medicação, podem ocorrer oscilações nas funções motoras, incitando maior vulnerabilidade aos acidentes por quedas. $\mathrm{O}$ idoso que cai pode apresentar aumento da dependência para as atividades de vida diária (AVD), redução da autonomia, perda da qualidade de vida, institucionalização, isolamento social, perda de mobilidade e até mesmo morte ${ }^{(8-9)}$.

Como consequências, há incidência das quedas, que são consideradas problema de saúde pública no Brasil e no mundo. O Sistema Único de Saúde (SUS) gasta a cada ano mais de $\mathrm{R} \$ 51$ milhões com o tratamento de fraturas decorrentes de queda ${ }^{(10)}$. Em estudo ecológico com componente de série temporal de 2008-2012, com dados obtidos do Sistema de Informações Hospitalares do SUS (SIH-SUS), foi registrada a ocorrência de 181 mil casos de fraturas de fêmur em pessoas com idade $\geq 60$ anos, sendo a média de 36.200 casos por ano, com ônus para o setor de saúde de $\mathrm{R} \$ 58,6$ milhões em internações ${ }^{(10-11)}$.

Considerando o ônus financeiro ao SUS, bem como psicológico aos idosos e familiares, faz-se necessário adotar estratégias de promoção da saúde e prevenção de agravos visando evitar as quedas. Nesse aspecto, as gerontotecnologias podem trazer inúmeros benefícios ao processo de viver humano, em especial, para o cuidado em saúde, pois a aplicação dessas estratégias configura-se como ferramenta de práticas educativas, prevenção de comorbidades e melhoria da qualidade de vida do idoso ${ }^{(12-13)}$.

O uso das gerontotecnologias no contexto da educação em saúde e da enfermagem fomenta e amplia as ações desempenhadas pelo enfermeiro na sua relação de cuidado com a pessoa idosa com a DP. Estudos apontam que as gerontotecnologias podem incutir inúmeros benefícios no processo do cuidado, já que contribuem com a educação em saúde, vislumbram novos olhares no processo de ensino aprendizagem, mediadas por interações pelo enfermeiro (locutor), idoso e familiares (leitor) e o material educativo (objeto do discurso). Dessa forma, a efetivação das gerontotecnologias tem o potencial de esclarecer aos pacientes/familiares/cuidadores sobre a doença e sua evolução clínica e, ainda, orientá-los sobre como exercer o cuidado ${ }^{(12-13)}$.

Para garantir sua efetividade, as tecnologias educacionais necessitam ser avaliadas, a fim de aumentar sua eficácia e adequabilidade para os que farão uso delas. A avaliação é ferramenta fundamental para os pesquisadores e profissionais na produção e utilização de tecnologias em saúde. Trata-se de tecnologias que, quando adequadas, proporcionam maior segurança ao implementar as ações nas práticas de cuidado ${ }^{(14)}$.

Diante do exposto, o objetivo do presente estudo foi avaliar a contribuição das gerontotecnologias, jogos da memória e cartilha, no cuidado de enfermagem ao idoso com DP, visando à prevenção de quedas. As tecnologias a serem avaliadas foram desenvolvidas por uma etapa anterior, em número de três, sendo confeccionadas a partir de uma dissertação de mestrado intitulada "Gerontotecnologias Educativas para o Idoso com Doença de Parkinson: promoção da saúde para prevenção de quedas”(15).

\section{MÉTODO}

Trata-se de uma pesquisa qualitativa, utilizando os princípios da Pesquisa Convergente-Assistencial (PCA). A PCA foi escolhida porque, durante o processo de construção e aplicação, mantém estreita ligação com a prática assistencial, com objetivo de resolver problemas, realizar mudanças e/ou introduzir inovações na situação da prática assistencial ${ }^{(16)}$.

O local do estudo foi um Grupo de Ajuda Mútua às Pessoas com a Doença de Parkinson (GAM Parkinson), localizado no litoral do estado de Santa Catarina. Os participantes da pesquisa foram nove idosos que frequentavam o GAM Parkinson e participaram da elaboração das gerontotecnologias desenvolvidas durante os encontros do grupo, além de dez profissionais titulados pela Sociedade Brasileira de Geriatria e Gerontologia (SBGG), listados no site da referida instituição (nomeados neste trabalho como juízes), que realizaram avaliação de conteúdo e legibilidade.

Foram utilizados os seguintes critérios de inclusão para os participantes juízes: ser titulado em gerontologia e cadastrado no site da SBGG, ter contato de e-mail disponível nas bases eletrônicas Google, Scientific Electronic Library 
Online (SciELO) e Scopus. Como critérios de exclusão, estabeleceram-se os seguintes critérios: membros da SBGG titulados em gerontologia que não estavam listados no site da mesma, titulados cujos contatos de e-mail não estavam disponíveis nas bases eletrônicas.

Foram incluídos idosos membros do GAM Parkinson, com participação nas reuniões há pelos seis meses e que aceitaram participar do estudo. Entre os critérios de exclusão, estavam idosos com score abaixo de quatro no teste do relógio e não diagnosticados com DP.

Todos os juízes selecionados foram convidados a participar do estudo, mediante duas cartas-convite via e-mail. O período de coleta de dados foi de agosto a outubro de 2017. Foram selecionados 169 titulados pela SBGG, dos quais 112 e-mails foram encontrados nas bases eletrônicas. Todos foram convidados individualmente, por meio de contato eletrônico em duas tentativas, sendo que a segunda ocorreu 15 dias depois da primeira tentativa. Dos 112, 20 responderam à pesquisa e dez aceitaram participar. Foi aplicado questionário de caracterização sociodemográfica dos juízes, destacando as variáveis sexo, idade e profissão.

Em relação às gerontotecnologias desenvolvidas e avaliadas nessa etapa do estudo, a primeira foi uma cartilha educativa "se liga na queda", que apresentou os seguintes aspectos: informações/conceitos sobre a DP e quedas; atividades interativas, cuidados e a importância da família e do cuidador sobre prevenção de quedas; no processo, além de jogos de memorização.

A segunda gerontotecnologia avaliada foi um jogo da memória, intitulado "não cai istepô". O nome do jogo foi escolhido como uma homenagem aos nativos, que usam tal expressão na cidade onde se desenvolveu o estudo. O jogo foi elaborado com base na marcha e na memória, identificadas como necessidades emergentes na avaliação realizada junto aos idosos com a DP estudados. Para tanto, utilizou-se material reaproveitado, disquetes antigos, que foram revestidos em EVA, e, em seguida, foram fixadas imagens produzidas por designer gráfico relacionadas à $\mathrm{DP}$ e à prevenção de quedas. O jogo finalizado apresentou 36 peças, sendo 18 pares de imagens que levam o idoso a refletir sobre a prevenção de quedas.

A terceira gerontotecnologia avaliada foi o jogo da memória "escorregou de maduro", cuja proposta do nome do jogo foi dada pelos próprios idosos, ao recordarem-se da expressão "caiu de maduro". O jogo é composto de 36 compact discs (CD) antigos revestidos com EVA (material reaproveitado), dentre os quais $18 \mathrm{CD}$ contêm apenas imagens, visando a prevenção de quedas, e $18 \mathrm{CD}$ apresentam imagens e condutas relacionadas à promoção da saúde para evitar quedas - nesse jogo, o objetivo do jogador é encontrar a peça correlacionada à imagem e aprender uma nova conduta para evitar quedas.

A avaliação dos juízes foi baseada nas orientações do "Guide to Creating and Evaluating Patient Materials"(9), que favorece a compreensão do sujeito do estudo e melhora sua capacidade de adesão à prevenção, ao tratamento. Para tanto, foi utilizado o instrumento "Suitability Assessment of
Materiais" (SAM), instrumento traduzido e adaptado para o português ${ }^{(17)}$ e Instrumento de avaliação da gerontotecnologia elaborado na dissertação ${ }^{(15)}$.

O Instrumento de avaliação da gerontotecnologia continha a avaliação do material quanto a exatidão científica, conteúdo, apresentação literária, ilustrações, material compreensivo, legibilidade e características da impressão do material educativo, qualidade da informação e espaço para opiniões pessoais $^{(15)}$. Já o SAM é um instrumento americano que se apresenta em forma de checklist com seis categorias, sendo 22 itens, possuindo escala de pontuação de zero a dois. Deve ser aplicado após a leitura do texto, sendo que dois considera-se o material superior (excelente), um adequado e zero não adequado, conforme os critérios objetivos incluídos no instrumento de avaliação da tecnologia educacional: conteúdo, compreensão do texto, ilustração, apresentação, motivação e adaptação cultural ${ }^{(17)}$.

Os dados foram inseridos no programa Microsoft Excel 2010, organizados em tabela e quadros, acrescentando junto às pontuações de cada item dos instrumentos as sugestões e opiniões dos juízes, sendo analisados os scores de cada instrumento e seguido as recomendações dos juízes, para reformulação dos materiais propostos. Posteriormente, foi realizada a interpretação pelos Índice de Legibilidade de Flesch-Kincaide (ILFK) e Índice de Facilidade de Leitura de Flesh (IFLF) em ferramenta própria do Microsoft Word 2010, ao instrumento de avaliação da gerontotecnologia, a fim de avaliar leitura da gerontotecnologia ${ }^{(18)}$.

Optou-se pelo índice de legibilidade, já que é considerado indicador de compreensão e facilidade de leitura, avaliando que, quanto maior o tamanho das palavras e frases, mais complexa será a leitura para o idoso. Por se tratar de idosos brasileiros, foi relevante para o estudo considerar o seu nível educacional, pois menos de 30\% têm nível superior ou secundário ${ }^{(19-22)}$.

O IFLF avaliou o grau de legibilidade dos textos na escala porcentual de 0 a 100 , enquanto o ILFK utiliza os valores de 0 a 35 e considera anos escolaridade exigidos para se compreender um determinado texto. $\mathrm{O}$ valor na escala porcentual é proporcional à facilidade de leitura, sendo que se preconiza que os percentuais fiquem entre 60 e $70 \%$. Para esta pesquisa, foi considerado que as tecnologias educacionais deveriam apresentar score acima de $70 \%$ como aceitável ${ }^{(19-21)}$.

Para tanto, seguiram-se suas respectivas fórmulas, sendo: para o ILFK $=((0,39 \times$ média de palavras por frase $)+(11,8$ x média de sílabas por palavra)) - 15,59. Para IFLF, usou-se: IFLF $=206,835-((1,015 \times$ comprimento médio da frase $)$ + 0,846 x (número de sílabas por 100 palavras)). Por fim, todos os textos foram selecionados e avaliados pelo Revisor Gramatical Automático para o Português, ferramenta de análise de textos do próprio Microsoft Office Word 2010, que avaliou tanto o IFLF quanto o ILFK ${ }^{(23-24)}$.

$\mathrm{O}$ presente estudo seguiu as diretrizes e normas regulamentadoras de pesquisas com seres humanos estabelecidas pela Resolução n ${ }^{\circ}$ 466/2012. Parecer foi emitido pela Universidade Federal de Santa Catarina, CAAE n ${ }^{\circ}$ 24349813.9.0000.0121. 


\section{RESULTADOS}

Dez participantes juízes avaliaram as gerontotecnologias, sendo quatro do sexo masculino e seis do sexo feminino, com idade variando de 36 a 76 anos. Quanto às formações, foram três fisioterapeutas, três assistentes sociais, dois enfermeiros e dois psicólogos. Quanto à área de atuação, seis atuavam como docentes, todos possuíam experiência com idosos com uma média de 17,6 anos, todos eram membros e titulados em gerontologia pela SBGG e todos tinham experiência em avaliação de materiais educativos. Além disso, todos tinham publicações Geriatria/Gerontologia, Elaboração/ Validação de material educativo, acessibilidade, bem como todos contavam com autoria em artigos científicos com temáticas relativas à Saúde do idoso/ Gerontologia/Geriatria publicados em periódicos classificados pela Coordenação de Aperfeiçoamento de Pessoal de Nível Superior (CAPES).

O resultado do SAM na somatória dos pontos atribuídos a cada item do instrumento categorizou a gerontotecnologia quanto à adequação do material educativo para os idosos. O cálculo foi realizado por meio do somatório total dos escores, dividido pelo total de itens do questionário. Para termos de avaliação, foi considerado o índice de concordância de $70 \%$ entre os juízes, considerando, assim, o material avaliado com o instrumento como excelente ou adequado, com destaque para leitura simples e objetiva, ilustrações do dia a dia e orientações específicas para o público-alvo.

Ao término da avaliação do SAM, foi utilizado instrumento de avaliação da gerontotecnologia, que contava com 47 itens e três questões abertas, cujo objetivo foi classificar a tecnologia educacional em consonância com o valor que se adequava à opinião do juiz, de acordo com a seguinte valoração: 1 Discordo totalmente, 2 Concordo com alguns aspectos do enunciado, 3 Concordo com a maior parte do enunciado, 4 Concordo totalmente, N/A não se aplica.

De acordo com relatos dos juízes e com base nos dados obtidos dos instrumentos das gerontotecnologias, estas são ferramentas eficazes para o ensino-aprendizagem do idoso com DP. A interpretação realizada a partir dos índices de ILFK e IFLF obteve recomendação da necessidade de seis anos de estudos para compreensão do material proposto no ILFK. Já a interpretação do IFLF considerou que o material apresentou $85 \%$ na facilidade para a leitura, concluindo-se ser adequado para os idosos.

A avaliação de conteúdo realizada pelos juízes foi positiva para a cartilha e os jogos da memória. Indicaram o material como excelente, ponderando acréscimo para as orientações práticas, principalmente pela forma lúdica e interativa do material. Essas tecnologias educacionais, por serem materiais de fácil compreensão, podem proporcionar ao idoso, ao utilizar o material, motivação em seguir as orientações propostas. Além disso, acredita-se que, pelo fato da construção dessas gerontotecnologias aqui apresentadas serem advindas de um processo de pesquisa que buscou problematizar as necessidades dos idosos e de profissionais na prevenção do evento quedas, este estudo alcançou seu público-alvo, conforme pode ser constatado no quadro síntese dos resultados na sequência.
Quadro 1 - Índices de avaliação de conteúdo e elegibilidade da gerontotecnologia educacional, segundo juízes.

\begin{tabular}{|l|c|c|c|}
\hline & SAM & ILFK & IFLF \\
\hline Avaliação juízes & $70 \%$ & 6 anos de estudo & $85 \%$ \\
\hline
\end{tabular}

Nota: SAM: Suitability Assessment of Materiais; ILFK: Índice de Legibilidade de Flesch-Kincaide; IFLF: Índice de Facilidade de Leitura de Flesh.

Fonte: Autoras (2020).

\section{DISCUSSÃO}

De acordo com o HelpAge Internationa ${ }^{(22)}$, o Brasil ocupa o $56^{\circ}$ lugar do ranking global que vai de 1-96 sobre avaliação do bem-estar social e econômico dos idosos. Os principais fatores relacionados são baixos índices de segurança, insatisfação com transporte público e falta de escolaridade. Este dado foi evidenciado na pesquisa, na qual apenas dois idosos apresentavam curso superior. Tal dado é de extrema importância para avaliação e desenvolvimento da gerontotecnologia adaptada ao nível de escolaridade da maioria dos idosos.

Nas gerontotecnologias, visando a promoção da saúde do idoso e a prevenção de agravos, o foco deve ser no desenvolvimento de tecnologias que irão contribuir para o cuidado gerontológico, com vistas à promoção da saúde, capacitando os indivíduos para que se tornem protagonistas em seu processo de cuidado e desenvolvam habilidades pessoais para mudança de conduta diante dos fatos de seu cotidiano ${ }^{(13,25)}$. Refletir sobre o cuidado de enfermagem sob o prisma tecnológico possibilita ao enfermeiro vislumbrar novas possibilidades de cuidado humanizado, inovador, capaz de transformar o cotidiano, melhorar a qualidade de vida do idoso, assim como seu bem-estar físico-psíquico-emocional, promovendo saúde ao prevenir quedas ${ }^{(25-26)}$.

Contudo, para a efetividade da gerontotecnologia, há a necessidade de sua avaliação. Neste estudo, a avaliação dos juízes revelou que as tecnologias educacionais são materiais educativos de conteúdo e válidas para prevenção de quedas em idosos com DP, com aparência atrativa e motivadora para jogar e/ou ler. Apesar do resultado, no ILFK, da necessidade de os idosos possuírem seis anos de estudos para utilização desses materiais e de $85 \%$ de facilidade na leitura, como indicado pelo IFLF, o material foi avaliado como adequado para os idosos.

Evidencia-se o interesse e a necessidade dos profissionais que atuam na gerontologia em utilizar as tecnologias educacionais ${ }^{(27)}$. Considera-se que as demandas estão mudando e rompendo paradigmas, como sugeriram os juízes, visto que as gerontotecnologias vão ao encontro de tal desafio, sendo um campo interdisciplinar que cada vez mais ganha espaço desde a sociologia até a enfermagem, com o objetivo de estimular seu uso e melhorar a qualidade das pessoas ${ }^{(2)}$.

Destaca-se que a prevenção de quedas deve ponderar o estilo de vida e o ambiente dos idosos, tendo em vista que cada idoso vivencia seu processo de cuidado de forma diferente e os riscos são diferentes aos idosos com a DP. Quando esse fator é negligenciado pelos profissionais de saúde e até mesmo familiares/cuidadores, os resultados tornam-se pequenos ${ }^{(28)}$. 
Dessa forma, ao trabalhar a promoção da saúde e o incentivo ao desenvolvimento de habilidade pessoais, é possível auxiliar no processo de empoderamento do idoso, atuando no seu processo de cuidado e proporcionando-lhe a oportunidade de focar em objetivos definidos e levá-lo a resultados efetivos na prevenção das quedas. O olhar sobre uma proposta das atividades educativas perpassa a temática de transmissão de informações, pois rompe com o paradigma do tratamento do idoso centrado na doença, já que visa a melhoria na qualidade de vida e não somente o aumento da estimativa de vida ${ }^{(29)}$.

Esse empoderamento favorece um aprendizado horizontal, no qual cada sujeito possui um conhecimento diferente e todos são igualmente valorizados. Auxilia, no idoso com $\mathrm{DP}$, o desenvolvimento de consciência crítica, sendo que o produto da gerontotecnologia vai além do cuidado tradicional, como o simples repasse de informação ${ }^{(28,30)}$. Além disso, as gerontotecnologias são ferramentas contributivas para auxiliar no cuidado realizado por familiares e cuidadores: facilitam o processo de ensino-aprendizagem entre os envolvidos; favorecem a troca de conhecimentos; constroem novos conhecimentos por meio do saber do outro; incentivam a socialização do idoso com DP; fortalece o vínculo entre todos os envolvidos, repercutindo na melhora do cuidado recebido e na adesão do idoso para prevenção de quedas ${ }^{(25)}$.

Dessa maneira, esta pesquisa recomenda que a enfermagem siga investindo no cuidado gerontológico, focada nas tecnologias educacionais, as quais necessitam ser construídas em conjunto com os idosos, com objetivo de esclarecer suas dúvidas e servir de alicerce para familiares/cuidadores. Para tanto, é fundamental que sejam embasadas nos preceitos interdisciplinares das gerontotecnologias, associando diferentes saberes em busca do ápice do cuidado gerontológico: o idoso com DP vivendo com qualidade, dignidade e, por consequência, melhorando seu estado geral de saúde e prevenindo quedas ${ }^{(25)}$.

Ponderando a complexidade em constituir um grupo de juízes renomados e titulados por instituição reconhecida nacional e internacionalmente, a SBGG, aliada à demanda de atividades desses profissionais, destaca a baixa adesão dos titulados para atender as solicitações de avaliação deste estudo. Em muitos casos, não se obteve sucesso na comunicação virtual, em outros, seus contatos não estavam disponíveis nas bases eletrônicas, outros não possuíam tempo hábil para participar do estudo, considerando a limitação do curto espaço de tempo para conclusão da pesquisa.

Somando a isso, considera-se como limitação a diversidade da população idosa brasileira. Desse modo, o material elaborado está adequado ao público participante do estudo, sendo que possivelmente precisará de alterações para tornar-se adequado aos idosos de outros contextos. Além disso, os idosos que apresentam redução da acuidade visual podem ter dificuldade de ler, sendo sugerido aumentar o tamanho da letra para melhor visualização.

\section{CONCLUSÃO}

As gerontotecnologias educacionais desenvolvidas têm conteúdo relevante, podendo ser utilizadas pelos idosos, familiares e cuidadores, a fim de gerar esclarecimento de questões sobre a temática prevenção de quedas para os idosos com DP. Desse modo, incluem os seguintes elementos: 1) cartilha educativa "se liga na queda", que tem informações/conceitos sobre a DP e quedas, atividades interativas, cuidados e a importância da família e do cuidador sobre prevenção de quedas, no processo, além de jogos de memorização; 2) jogo da memória "não cai istepô", que apresenta nome como homenagem aos nativos da região do estudo, sendo elaborado com base na marcha e memória, identificadas como necessidades emergentes na avaliação realizada junto aos idosos com a DP estudados, o qual utiliza material reaproveitado (disquetes antigos, revestidos em EVA, com imagens produzidas por designer gráfico relacionadas à $\mathrm{DP}$ e prevenção de quedas fixadas), com 36 peças, sendo 18 pares de imagens que levam o idoso a refletir sobre a prevenção de quedas; 3) jogo da memória "escorregou de maduro", cuja proposta do nome desse jogo foi dada pelos próprios idosos ao recordarem-se da expressão "caiu de maduro", é composto de 36 peças produzidas de forma sustentável (CD antigos revestidos com EVA), dentre os quais $18 \mathrm{CD}$ contêm apenas imagens, visando a prevenção de quedas, e $18 \mathrm{CD}$ apresentam imagens e condutas relacionadas à promoção da saúde para evitar quedas, com o objetivo de encontrar a peça correlacionada à imagem e aprender uma nova conduta para evitar quedas.

Comprovou-se a efetividade das gerontotecnologias educacionais para os idosos com DP, principalmente quanto ao incentivo para prevenção de quedas, autocuidado, o qual esclarece dúvidas por meio do fornecimento de conhecimentos sobre a DP, sinais e sintomas, trazendo novos conceitos e cuidados para evitar as quedas. Essas tecnologias elaboradas na forma de material educativo apresentam importância fundamental para prevenção de quedas, visando à promoção da saúde. Por meio da utilização de atividades lúcidas e de fácil compreensão, tornam-se mais atrativas para adquirir informações/conhecimentos, sensibilizando para mudança de hábitos e/ou condutas, além de incentivar o autocuidado.

Destaca-se, como contribuição e implicação deste estudo, o estímulo ao cuidado de enfermagem utilizando os preceitos das tecnologias educacionais como metodologias contributivas aos profissionais da enfermagem. Visa-se fornecer possibilidades inovadoras e instigantes ao desenvolvimento de habilidades pessoais, rompendo com os paradigmas do cuidado de enfermagem e a relação enfermeiro-cliente.

As gerontotecnologias podem ser utilizadas como instrumentos que complementam as atividades dos profissionais de saúde, direcionadas ao ensino e à prevenção de quedas, além de esclarecer a fisiopatologia da DP. Além disso, podem ser utilizadas para e por cuidadores e familiares, esclarecendo dúvidas e servindo de ferramenta de apoio, de modo a fundamentar o processo do cuidado. 
RESUMO

Objetivo: Avaliar a contribuição de gerontotecnologias no cuidado gerontológico de enfermagem ao idoso com Doença de Parkinson, visando à prevenção de quedas. Método: Pesquisa Convergente Assistencial, mediante a construção e a avaliação de gerontotecnologias focadas na prevenção de quedas em idosos com Doença de Parkinson. Realizaram-se avaliação clínica, entrevistas e oficinas com idosos, bem como análise e julgamento dos materiais desenvolvidos por dez juízes titulados em gerontologia pela Sociedade Brasileira de Geriatria e Gerontologia. Resultados: Os idosos necessitam de no mínimo seis anos de estudo para compreender a gerontotecnologia educacional desenvolvida. Os objetivos relacionados a conteúdo, compreensão do texto, ilustração, apresentação, motivação e adaptação cultural foram alcançados. Conclusão: A gerontotecnologia educacional desenvolvida tem conteúdo relevante, podendo ser utilizada pelos idosos, familiares e cuidadores, a fim de gerar esclarecimento de questões sobre a temática prevenção de quedas para os idosos com Doença de Parkinson.

\section{DESCRITORES}

Multimídia; Cuidados de Enfermagem; Idoso; Doença de Parkinson.

\section{RESUMEN}

Objetivo: Evaluar la contribución de las gerontecnologías en los cuidados de la enfermería gerontológica de los adultos mayores con Parkinson, con el objetivo de prevenir las caídas. Método: Se trata de una Investigación Convergente Asistencial, a través de la construcción y evaluación de gerontecnologías enfocadas en la prevención de caídas de adultos mayores con Parkinson. Se llevó a cabo una evaluación clínica, además de entrevistas y talleres con los adultos mayores, así como el análisis y el juicio de los materiales desarrollados por diez jueces graduados en gerontología por la Sociedad Brasileña de Geriatría y Gerontología. Resultados: Las personas mayores necesitan al menos seis años de estudio para comprender la gerontecnología educativa. Se cumplieron los objetivos relacionados con el contenido, la comprensión del texto, la ilustración, la presentación, la motivación y la adaptación cultural. Conclusión: La gerontología educativa desarrollada tiene un contenido relevante que puede ser utilizado por los mayores, los familiares y los cuidadores con el fin de obtener claridad en el tema de la prevención de caídas de los adultos mayores con la enfermedad de Parkinson.

\section{DESCRIPTORES}

Multimedia; Atención de Enfermería; Anciano; Enfermedad de Parkinson.

\section{REFERÊNCIAS}

1. Fozard JL, Rietesema J, Bouma H, Graafmans JAM. Gerotechnology: creating enabling environments for the challenges and opportunities of aging. Educ Gerontol. 2000;26(4):331-44. https://doi.org/10.1080/036012700407820

2. Rodeschini G. Gerotechnology: a new kind of care for aging? An analysis of the relationship between older people and technology. Nurs Health Sci. 2011;13(4):521-8. https://doi.org/10.1111/j.1442-2018.2011.00634.x

3. Romani JM, Mourão LC, Almeida ACV. The pedagogical practice of the nurse from the emergency unit of the regional polyclinic of engenhoca: barriers, conquests and challenges. J Nurs UFPE on line 2017;11(5):1963-65. https://doi.org/10.5205/1981-8963v11i5a23347p1963-1965-2017

4. World Heath Organization. Ageing and life-course. Elder abuse [Internet]. Geneva: World Health Organization; 2019 [cited 2019 Aug 29]. Available from: https://www.who.int/ageing/projects/elder_abuse/en/

5. Bloom ED, Luca LD. The global demography of aging: facts, explanations, future [Internet]. Born: IZA; 2016 [cited 2020 Feb 12]. (Discussion paper, No 10163). Available from: http://ftp.iza.org/dp10163.pdf

6. Kanasi E, Ayilavarapu S, Jones J. The aging population: demographics and the biology of aging. Periodontol 2000. 2016;72(1):13-8. https:// doi.org/10.1111/prd.12126

7. Freitas EV, Py L. Tratado de geriatria e gerontologia. 4th ed. Rio de Janeiro: Guanabara Koogan; 2016.

8. Falsarella GR, Gasparotto LP, Coimbra AM. [Falls: concepts, frequency and applications to the elderly assistance. Review of the literature]. Rev Bras Geriatr Gerontol. 2014;17(4):897-910. Portuguese. https://doi.org/10.1590/1809-9823.2014.13064

9. American Geriatrics Society, British Geriatrics Society. AGS/BGS Clinical practice guideline: for prevention of falls in older persons [Internet]. New York: American Geriatrics Society; 2010 [cited 2017 Oct 7]. Available from: http://www.americangeriatrics.org/health_ care_professionals/clinical_practice/clinical_guidelines_recommendations/2010/

10. Brasil. Ministério da Saúde. Portal Brasil. Quedas. Tombos quase sempre são sinais de que o idoso está com algum problema de saúde [Internet]. Brasília, DF: Ministério da Saúde; 2012 [cited 2017 Oct 7]. Available from: http://www.brasil.gov.br/saude/2012/04/quedas

11. Soares DS, Mello LM, Silva AS, Martinez EZ, Nunes AA. [Femoral fractures in elderly Brazilians: a spatial and temporal analysis from 2008 to 2012]. Cad Saude Publica. 2014;30(12):2669-78. Portuguese. https://doi.org/10.1590/0102-311x00218113

12. Dias GMS, Labegalini CMG, Moura MB, Silva IVTC, Carreira L, Baldissera VDA. [Participatory construction of a gerontechnology on stroke]. Enfermagem Brasil 2018;17(5):436-443. Portuguese. https://doi.org/10.33233/eb.v17i5.1184

13. Barros EJ, Santos SS, Gomes GC, Erdmann AL. [Educational geronto-technology for ostomized seniors from a complexity perspective]. Rev Gaúcha Enferm. 2012;33(2):95-101. Portuguese. https://doi.org/10.1590/S1983-14472012000200014

14. Oliveira SC, Lopes MVO, Fernandes AFC. Development and validation of an educational booklet for healthy eating during pregnancy. Rev Latino-Am Enfermagem. 2014;22(4):611-20. https://doi.org/10.1590/0104-1169.3313.2459

15. Ferreira JM. Gerontotecnologias educativas para o Idoso com Doença de Parkinson: Promoção da Saúde para Prevenção de Quedas [Dissertation]. Florianópolis: Universidade Federal de Santa Catarina; 2017.

16. Trentini, M.; Paim, L; Silva, D.M.G.V. Pesquisa convergente assistencial: delineamento provocador de mudanças nas práticas de saúde. 3th ed. Florianópolis: Moria; 2014.

17. Sousa CS, Turrini RNT, Poveda VB. Translation and adaptation of the instrument "Suitability Assessment of Materials" (SAM) into portuguese. Rev Enferm UFPE on line. 2015;9(5):7854-61. https://doi.org/10.5205/reuol.6121-57155-1 
18. Doak CC, Doak LG, Root JH. Teaching patients with low literacy skills. 2nd ed. Phladelphia: JB Lippincott; 1996.

19. Flesh R. A new readability yardstick. J Appl Psychol. 1948;32(3):221-33. https://doi.org/10.1037/h0057532

20. Martins TBF, Ghiraldelo CM., Nunes MGV, Oliveira JON. Readability formulas applied to textbooks in Brazilian portuguese. São Carlos: ICMSC-USP; 1996.

21. Franco RC. [Assessment of Nutritional Guidance Manual for People with Type 2 diabetes mellitus] [thesies]. Ribeirão Preto: Universidade de São Paulo; 2015. Portuguese.

22. HelAge International. Global AgeWatch Index 2015: insight report [Internet]. 2015 [cited 2017 Nov 3]. Available from: https://reliefweb. int/sites/reliefweb.int/files/resources/global-agewatch-index-2015-insight-report.pdf

23. Flesch RF. Let's start with the formula. In: University of Canterbury. How to write plain English. 2nd ed. Canterbury. Barnes \& Noble; 2001.

24. Teles LM, Oliveira AS, Campos FC, Lima TM, Costa CC, Gomes LF, et al. Development and validating an educational booklet for childbirth companions. Rev Esc Enferm USP. 2014;48(6):977-84. https://doi.org/10.1590/S0080-623420140000700003

25. Ilha S, Santos SSC, Backes DS, Barros EJL, Pelzer MT, Oliveira AMN Educational and care-related (geronto) technology in Alzheimer's disease and in supporting the elderly/family: perspective of teachers and students. Rev Anna Nery. 2017;21(2):1-8. https://doi.org/10.5935/14148145.20170039

26. Pereira GN, Morsch P, Lopes DG, Trevisan MD, Ribeiro A, Navarro JH, et al. [Social and environmental factors associated with the occurrence of falls in the elderly]. Cien Saude Colet. 2013;18(12):3507-14. Portuguese. https://doi.org/10.1590/S1413-81232013001200007

27. Cordeiro LI, Lopes TO, Lira LEA, Feitoza SMS, Bessa MEP, Pereira MLD, et al. Validation of educational booklet for HIV/Aids prevention in older adults. Rev Bras Enferm. 2017;70(4):808-15. https://doi.org/10.1590/0034-7167-2017-0145

28. Schepens SL, Panzer V, Goldberg A. Randomized controlled trial comparing tailoring methods of multimedia-based fall prevention education for community-dwelling older adults. Am J Occup Ther. 2011;65(6):702-9. https://doi.org/10.5014/ajot.2011.001180

29. Castro APR, Vidal ECF, Saraiva ARB, Arnaldo SM, Borges AMM, Almeida MI. Promoting health among the elderly: actions in primary health care. Rev Bras Geriatr Gerontol. 2018;21(2):155-63. https://doi.org/10.1590/1981-22562018021.170133

30. Hammerschmidt KSA, Lenardt MH. [Innovative educational technology for empowering the elderly with diabetes mellitus]. Texto Contexto Enferm. 2010;19(2):358-65. Portuguese. https://doi.org/10.1590/S0104-07072010000200018 